\title{
The Fatigue Life Analysis of the Battery Bracket
}

\author{
Meishi zhou ${ }^{1, \mathrm{a}}$, Huaixian Yin ${ }^{2, \mathrm{~b}}$,Tiezhu Zhang ${ }^{1, \mathrm{c}}$, Hongxin Zhang ${ }^{1, \mathrm{~d}}$, Gaojun Liu ${ }^{1, \mathrm{e}}$ \\ ${ }^{1}$ Mechanical and Electronic Engineering College, QingDao University, Qingdao 266071, China \\ ${ }^{2}$ Scientific Research Office, QingDao University, Qingdao 266071, China \\ ªdzhoums@163.com, byhx6279010@163.com,,qqdzhangtz@163.com,,qduzhx@126.com,
}

Key words: road spectrum; battery bracket of electric bus; fatigue analysis

Abstract: To verify the battery bracket fatigue life of electric bus, the finite element model of the battery bracket is built, and the equivalent stress distribution of the frame was got. Multi-body dynamics vehicle model was built in ADAMS/Car. The simulation analysis was conducted based on the road spectrum compiled by MATLAB to simulate $\mathrm{C}$ level road model. The maximum vertical acceleration of electric car in the road is obtained. The load of the battery bracket is calculated. So as to get the finite element solution. Combined with the random vibration analysis and the results of finite element analysis, the fatigue life of the battery bracket is calculated by combing the material S-N curve. Finally, the battery bracket was evaluated comprehensively according to the strength,stiffness, and cumulative fatigue usage. The results could provide a theoretical basis for future optimization.

\section{Introduction}

From 1980s, with the attention of the quality of air and the greenhouse effect, the study of electric vehicles is unprecedented enthusiasm.By now, The research of electric vehicle is becoming more and more mature by the world's major automobile enterprise groups, such as GM, Nissan, Ford, Toyota and Honda, and so on. Nowadays, the most difficult technical difficulty for electric vehicle is the research of electric vehicle battery[1]. The battery bracket is a component of the parts of a battery mounted on an electric vehicle. So the static strength and fatigue strength are important to the electric vehicle driving reliability. The fatigue damage is more prominent than other parts, because of its complex structure and uneven force. So the battery bracket's durability test should be carried out in its developing process whose cost is higher and cycle is longer [2]. However, most problems appear after the completion of the product design that bring certain difficulty to the design change. Now today, we can make predictions for the durability of the battery bracket, find the weak link of the structure, put forward reasonable improvement program at the initial stage of product design through the finite element fatigue analysis [3]. What is more, we can also greatly reduce or eventually avoid the fatigue test.

The electric bus driving on the $\mathrm{C}$ grade road was simulated throng the multi-body dynamics bus model in this paper, and The maximum vertical acceleration of electric car in the road is obtained. The finite element model was built and static analysis was carried out . At last, the fatigue analysis was carried out to verify whether the frame structure is reasonable. The results could provide a base for future optimization.

\section{The establishment of hybrid bus model and road model}

The models of bus suspension, steering system, tire and body model were built with ADAMS/Car in 
accordance with the data provided by the certain enterprise. The multi-body dynamics bus model is finally obtained through the communication interface of relative assembly.

The main source of the vibration in the process of the hybrid bus driving was caused by the ground roughness. Therefore, the appropriate road model has a great influence on the accuracy of the simulation. In the main research of road model, there are two ways to get the right road model. The first method is through software to simulate the road and the second is through the actual measured experiment data processing to get the road spectrum.

The road spectrum was obtained by using the first method in this paper. The longitudinal roughness distribution of the $\mathrm{C}$ grade random road was simulated with MATLAB as shown in Figure 1. The road model was built by using Road Builder to compile the road in ADAMS/CAR. Hybrid bus model and road model is shown in Figure 2. The complete hybrid bus model and road model can be simulated.

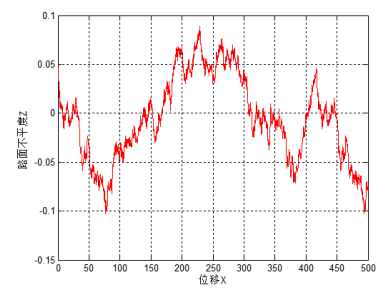

Fig.1 Road spectrum of C grade

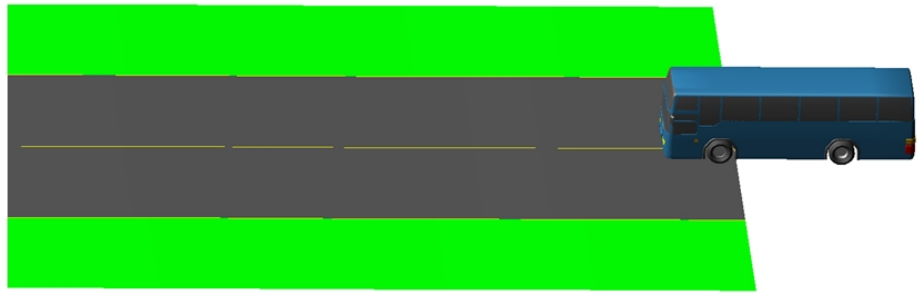

Fig.2 Hybrid bus model and road model

\section{Finite element transient dynamics analysis of the electric bus}

When the vehicle is traveling at a certain speed $v$ on the road with spatial frequency $n$, the time frequency $\mathrm{f}$ is the product of $\mathrm{v}$ and $\mathrm{n}$.

$$
f=n \times v
$$

In the formula $v=40 \mathrm{~km} / \mathrm{h}=11.11 \mathrm{~m} / \mathrm{s} . n$ takes the spatial reference frequency $n_{0} . n_{0}=0.1 \mathrm{~m}^{-1}$.

The simulation method is Straight-Line Event in the Full-Vehicle Analysis. The simulation time takes 30s. According to its maximum speed, the setting simulation speed is $40 \mathrm{~km} / \mathrm{h}$. After the dynamic analysis, The vehicle body vibration acceleration were obtained. as shown in Figure 3 .The maximum vertical acceleration of electric car in the road was $5.280867 \mathrm{e}-002 \mathrm{~g}, \mathrm{~g}=9.8 \mathrm{~m} / \mathrm{s}^{2}$, The maximum vertical acceleration of the car body is calculated

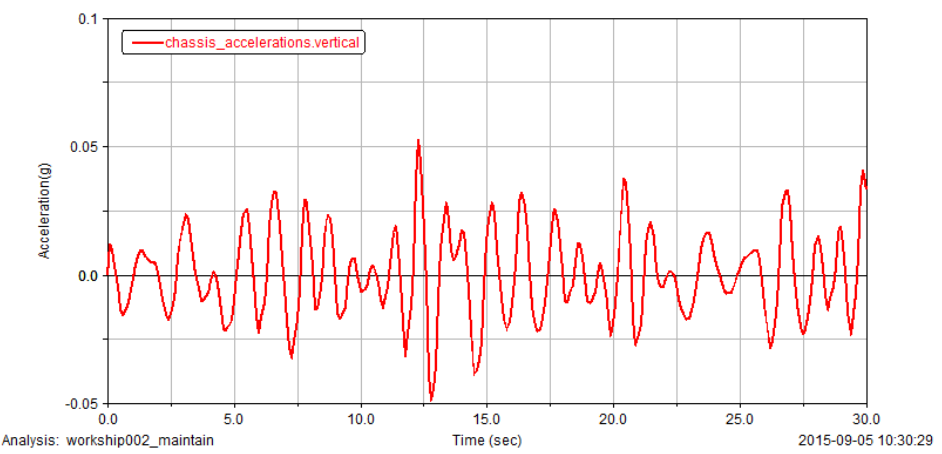

Fig.3The vehicle body vibration acceleration 


\section{Fatigue life analysis of the frame}

Fatigue is a phenomenon of the structure's bearing the repetitive load within the range of a certain load level which is less than the ultimate static load. The fatigue process is that the effect of repeated load leads to internal parts and materials damage and the greatest damage stress level is less than the material yield limit. Therefore, the static strength design can't reflect the actual loading conditions of the battery bracket which is loaded with symmetric fatigue load and alternating cyclic $\operatorname{load}[4]$.

There are various ways to obtain the structure stress and strain change, one of the best way is that the transient analysis was carried out with the loads and boundary conditions which vary with time to get the structure stress and strain for every moment. However, this method consumes too much computer resources. The simplified equivalent way was adopt in this paper. We need only obtain the distribution equivalent stress based on the upward node forceand the equivalent stress distribution based on downward node force. In other words, the dynamic loads were simplified as an alternating cyclic load.

The material of the battery bracket is PCABS. Reference to the relative papers, the EX of the material is $2.41 \times 10^{9} \mathrm{Mpa}$; the poisson distribution of the material is 0.39 ; the density of the material is $1.07 \times 10^{3} \mathrm{~kg} / \mathrm{m}^{3}$. The static analysis of the battery bracket was calculated based on ANSYS. The element type is SOLID187 and the mesh size was $0.01 \mathrm{~m}$. The constraints of the model is the bottom area of the battery bracket.

Consider battery box to withstand the most dangerous situation, when the battery thermal expansion of the lowermost support plate extrusion led to bear the total weight of five batteries, battery maximum equivalent stress analysis lowermost bracket shell to withstand the weight of five battery. Calculation of stress fatigue life of the battery box solution obtained by.

Battery weighs $1.5 \mathrm{~kg}$,total stent node N 144. Body maximum vertical acceleration a $=0.52 \mathrm{~m} / \mathrm{s}^{2}$. Battery holder under alternating loads by up nodal force $\mathrm{F} / \mathrm{N}=5 \mathrm{mg} / 2 \mathrm{~N}=0.2552 \mathrm{~N}$; nodal by downward force $\mathrm{F} / \mathrm{N}=5 \mathrm{~m}(\mathrm{~g}+\mathrm{a}) / 2 \mathrm{~N}=1.5 \times 5(9.8+0.52) / 2 \mathrm{~N}=0.26875 \mathrm{~N}$.

Fixed the bottom, applying downward force to get the battery case nodal maximum equivalent stress is 3.02Mpa. Its stress cloud shown in Figure 4.

Battery holder under alternating loads.Bearing upward nodal force $0.2552 \mathrm{~N}$, and bearing downward nodal force $0.26875 \mathrm{~N}$. As the boundary conditions on the battery holder finite element analysis, the battery holder equivalent stress distribution is shown in Figure 5.

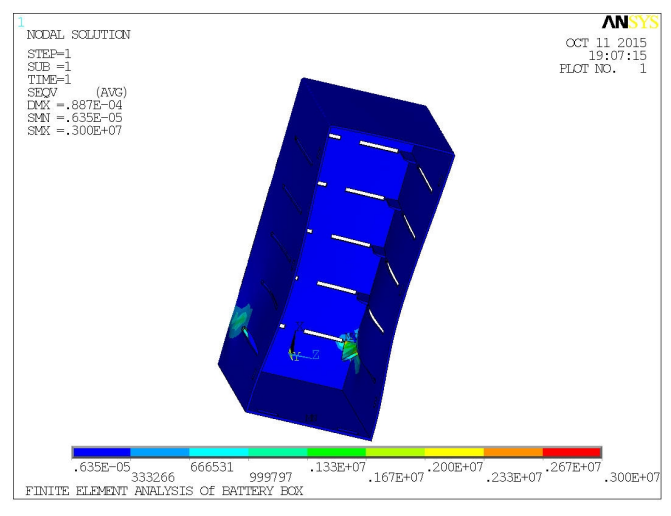

Fig.4 maximum stress based on downward force Fig.5 maximum stress based on upward force

Finite element analysis results show up on the battery holder nodal forces

The maximum equivalent stress with the downward load force as boundary condition was $\mathrm{MPa}$ which was far less than the material allowable stress, and the maximum displacement was 
$0.014 \mathrm{~mm}$, so the strength and stiffness could meet the requirements of the battery bracket. The maximum stress with the upward load force as boundary condition was MPa.refer to relative paper, the SN curve of the material is obtained as shown in Figure 6.

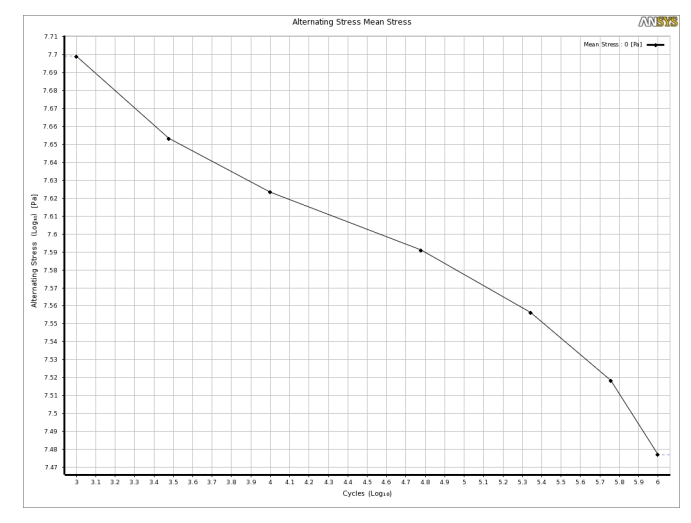

Fig.6 SN curve

According to the dynamic load analysis and the results of finite element analysis, the fatigue life of the battery bracket is calculated to obtain the specified location of fatigue life by combing the material S-N curve. The maximum equivalent stress position of the frame was selected as the specified location to calculate the frame fatigue life in this paper. The fatigue analysis result is shown in Figure 7.

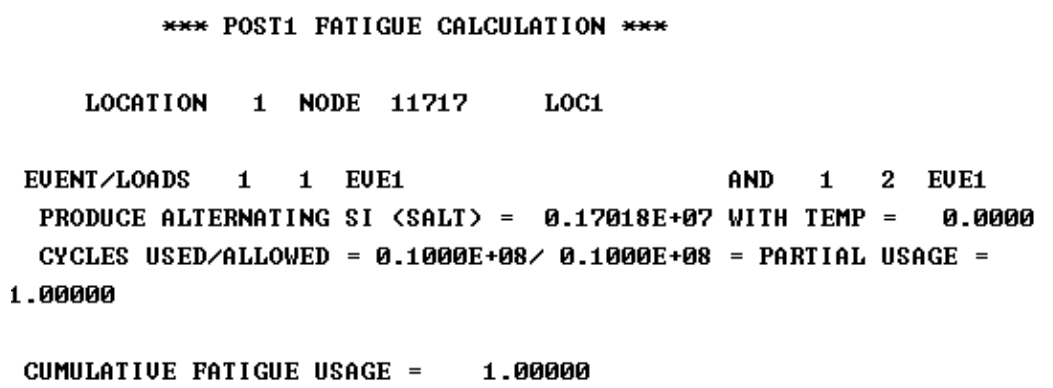

Fig.7 Fatigue analysis results

This battery bracket business requirements with unlimited fatigue life, calculated through finite element fatigue fatigue life of $10^{7}$. Generally believed that the fatigue life of $10^{7}$ is infinite life. So this battery bracket meets the requirements of the fatigue life.

\section{Conclusion}

Because of battery thermal expansion extrusion, the most bottom support plate supports the weight of five batteries. Consider the most dangerous working conditions, The maximum equivalent stress of the battery bracket was Mpa, which was far less than the material allowable stress, and the maximum displacement was $0.014 \mathrm{~mm}$, so the strength and stiffness could meet the requirements of the battery bracket. The fatigue life of the battery bracket is calculated. The results show that the allowable cycle number of the battery bracket is times while the expected cycle number is million times. At this time, the cumulative fatigue usage is 0.11294 that was less than 1 . So the battery bracket design was safe. The final results show that the battery bracket can satisfy the usage, and the fatigue strength of the battery bracket is surplus which can provide a theoretical basis for the optimization. 


\section{Acknowledgements}

The authors gratefully acknowledge the financial support from the Funded by the National High-tech R\&D Program(863Program) of China(No.2014AA052303), Provincial Shandong Province Foundation of China (No. 2014ZZCX05501 and No. 2014GGX103044).Cultivation of New and Strategical Industries of Qingdao(14-8-1-2-gx).

\section{Reference}

[1] Yang Qingquan, Gu Zhengqi, MI Chengji. Automotive Engineering. 2012,34(11),1016-1019.

[2] Huang Minfeng, Jiang Yingchun. Mechanical Research \& Application. 2008,21(2), 57-60.

[3] Gaojun Liu, Tiezhu Zhang, Hongxin Zhang: Applied Mechanics and Materials, Vols. 633-634(2014) pp1148-1153.

[4] Li Nan, Sun Huanwu, Yan Jie. Mechanical Engineering \& Automation. 2012, (5),9-11. 